\title{
Etiologies and characteristics of children with chief complaint of short stature
}

\author{
Kyung Chul Song, MD', \\ Song Lee Jin, MD', \\ Ah Reum Kwon, MD', \\ Hyun Wook Chae, MD', \\ Jung Min Ahn, MD', \\ Duk Hee Kim, MD, PhD², \\ Ho-Seong Kim, MD, PhD ${ }^{1}$
}

'Department of Pediatrics, Severance Children's Hospital, Yonsei University College of Medicine, Seoul, 'Sowha Chidren's Hospital, Seoul, Korea
Received: 26 September, 2014 Revised: 14 October, 2014 Accepted: 21 October, 2014

\footnotetext{
Address for correspondence: Ho-Seong Kim, MD, PhD

Department of Pediatrics, Severance Children's Hospital, Yonsei University College of Medicine, 50 Yonseiro, Seodaemun-gu, Seoul 120-752, Korea

Tel: +82-2-2228-2050

Fax: +82-2-393-9118

E-mail:kimho@yuhs.ac
}

Purpose: Short stature is a very common reason for visits to pediatric endocrine clinics. It could be the first sign of an underlying disease. The purpose of this study is to investigate the etiologies and general characteristics of subjects who visited an outpatient clinic due to short stature.

Methods: We retrospectively reviewed the medical records of 3,371 patients who visited Severance Children's Hospital with the chief complaint of short stature from 2010 to 2012. Medical history, auxological data, and laboratory tests including bone age were collected and analyzed. Chromosome studies or combined pituitary function tests were performed if needed.

Results: Approximately $89.4 \%$ of the subjects with the chief complaint of short stature who visited the outpatient clinic were of normal height, and only $10.6 \%$ of subjects were identified as having short stature. Of the subject of short stature, $44.7 \%$ were classified as having normal variant short stature; that is, familial short stature $(23.0 \%)$, constitutional delay in growth (17.7\%), and mixed form (3.9\%). Pathological short stature was found in 193 subjects (54.2\%). Among pathological short stature, most common etiology was growth hormone deficiency (GHD) (38.9\%).

Conclusion: A majority of children had a normal height. Among children with short stature, pathological short stature and normal variants occupied a similar percentage. GHD was the most common cause of pathological short stature and found in about $20 \%$ of the children with short stature. In pathological short stature, the height, height velocity, and IGF-1 level were lower than in normal variants.

Keywords: Short stature, Etiology, Growth

\section{Introduction}

Short stature is defined as a height below the third percentile or more than two standard deviations below the corresponding mean height for those of the same age, sex, and race. Short stature is one of the most common causes of referral to a pediatric endocrinologist. In fact, a large number of children have visited pediatric endocrinology clinic due to short stature. This trend is based on the belief that tall men are associated with the qualities expected of successful people ${ }^{1)}$. Previous studies reported that physical height and self-esteem correlated significantly, therefore, those in high-status jobs are generally believed to be taller than those in low-status jobs $^{2}$. Moreover, many reports indicated that children with short stature were academically and socially handicapped ${ }^{2-4)}$. In contrast, other studies suggested that the psychological adaptation of individuals who were shorter than average was largely indistinguishable from that of others ${ }^{5,6)}$. Despite there is no evidence that short stature is associated with psychosocial stress, psychological adaption, or academic performance, social stereotype of height, such as positive attributes being linked to tallness and negative attributes to shortness, still exist. Such popular stereotypical concepts about height in our society lead to hospital visits for short 
stature in children ${ }^{7)}$.

Short stature may be the first clinical manifestation of an underlying pathological disease, such as growth hormone deficiency (GHD), chronic diseases, or clinically defined syndromes; thus, a precise evaluation of short stature is crucial. Although most children with short stature are assessed as being healthy even after evaluation, careful monitoring of growth status (e.g., measurement of height, weight, and growth velocity) over time is essential for child healthcare. Short stature is a result of various etiologies, which are categorized as pathological causes and normal variants. Normal variant short stature consists of familial short stature (FSS) and constitutional growth delay (CGD), while pathological causes are subdivided into endocrine diseases, clinically defined syndromes, chronic diseases, metabolic diseases and others ${ }^{8)}$. Recently, clinicians have classified normal variant short stature as one type of idiopathic short stature (ISS), of which a diagnosis can be made after excluding the evidence of systemic, endocrine, nutritional, or chromosomal abnormalities. In addition, proper classification and precise diagnosis of short stature are essential to determine whether growth hormone $(\mathrm{GH})$ treatment is needed or not.

The aim of this study is to determine the etiological profile and distribution of the patients who visited an outpatient clinic to evaluate short stature.

\section{Materials and methods}

\section{Subjects}

This study is based on the retrospective analysis of the data of 3,664 children who were evaluated for short stature at the Department of Pediatrics, Severance Children's Hospital, Yonsei University College of Medicine, from January 2010 to December 2012. Patients who had a follow-up period less than 1 year were excluded. Accordingly, 293 patients were excluded and 3,371 patients were analyzed.

\section{Methods}

Short stature was defined as a height below the third percentile for the corresponding age and gender in a Korean growth chart that was revised in 2007, and etiological categorization was based on Ranke MB's classification ${ }^{8}$. We collected data on the patients' medical history (age, sex, intrauterine period, birth weight, yearly growth rate (YGR), midparental height (MPH), medication history, admission history, and outpatient history), physical findings (height, weight, upper/ lower ratio, Tanner staging, and any abnormal findings), and laboratory tests. Primary screening tests including complete blood count, routine chemistry, T3, free T4, thyroid-stimulating hormone, insulin-like growth factor-I (IGF-I), insulin-like growth factor binding protein-3 (IGFBP-3), and urine analysis were performed. Bone age was estimated through left hand $\mathrm{X}$-rays using the Greulich-Pyle method ${ }^{9)}$ performed by the same observer. Predicted adult height $(\mathrm{PAH})$ was estimated using the Bayley-Pinneau method ${ }^{10)}$. In subjects whose heights was below the third percentile for the same age and sex and growth velocity was attenuated, combined pituitary function tests and brain magnetic resonance imagings were performed. Additionally, chromosome studies were done in female patients with short stature below the third percentile.

FSS was attributed to a patient with a normal birth height and weight, a MPH below tenth percentile, a normal YGR, and a bone age appropriate for their chronologic age. CGD was attributed to a patient with a normal birth height and weight, a MPH above tenth percentile, a normal YGR, and a delayed bone age one to two years. GHD attributed to a patient whose $\mathrm{GH}$ levels were less than $10 \mathrm{ng} / \mathrm{mL}$ after stimulation in two kinds of $\mathrm{GH}$ provocation tests, such as insulin tolerance test and L-dopa test ${ }^{8}$. Diagnosis of ISS was made after evidence of systemic, endocrine, nutritional or genetic abnormalities was not found.

\section{Statistical analysis}

Statistical analysis of the results was performed using IBM SPSS Statistics ver. 20.0 (IBM Co., Armonk, NY, USA). All data was expressed as mean \pm standard deviation. Chi-square and $t$-test distributions were performed for between-pairs comparisons, and comparisons among groups were performed using analyses of variances. Differences were regarded as significant when $P<0.05$.

\section{Results}

\section{Frequency of children with chief complaints of short stature}

Among 3,371 subjects, 3,015 (89.4\%) had a normal height and $356(10.6 \%)$ had short stature below the third percentile in the growth chart (Table 1). Of those with short stature, 159 (44.7\%) were normal variants, 193 (54.2\%) had pathological short stature, and 4 patients (1.1\%) were uncategorized (Table 2). In the group of normal variants, there were 82 patients $(23.0 \%)$ with FSS, 63 patients (17.7\%) with CGD, and 14 patients (3.9\%) who had both. In the uncategorized group, 2 patients were suspected of having GHD because of attenuated growth and low levels of IGF-I and IGFBP-3 (patient 1: -0.6 SDS IGF-I, -1.3 SDS IGFBP-3; patient 2: -0.5 SDS IGF-I, -1.1 SDS IGFBP-3). While follow-ups without GH provocation tests, these cases were missing.

Fig. 1 showed the distribution curve of the children's height.

Table 1. Frequency of children with chief complaints of short stature

\begin{tabular}{lc}
\hline Category & No. of subjects (\%) \\
\hline Normal ( $>3$ percentile) & $3,015(89.4)$ \\
Short stature ( $<3$ percentile) & $356(10.6)$ \\
Total & $3,371(100)$ \\
\hline
\end{tabular}


Of the children with normal heights, 1,196 (39.7\%) had a SDS of -2 to -1 , and $1,015(33.7 \%)$ had a SDS of -1 to 0 . These findings suggest that most children with normal heights (73.3\%) who visited the hospital due to short stature had heights less than the average, with a SDS of -2 to 0 .

\section{Frequency of the various diagnoses of the children with pathological short stature}

Of the patients with pathological short stature, the most common cause was GHD (38.9\%), followed by ISS (23.2\%), small for gestational age (11.3\%), and Turner syndrome (9.3\%) (Table 3$)$. The others $(12.4 \%)$ were considered to have diverse etiologies, such as clinically defined syndromes, skeletal dysplasias, metabolic disorders, and chronic renal failures. GHD was diagnosed in approximately $20 \%$ of children with short stature.

\section{Causes of short stature in patients with GHD}

Of the patients with GHD, 21 patients (28.0\%) had morphological brain abnormalities or underlying diseases, and 54 patients (72.0\%) had no specific brain lesions (Table 4). Of the 21 patients with organic GHD, Rathke's cleft cyst (9.3\%) and hypoxic brain damage $(9.3 \%)$ were the most common causes,

Table 2. Frequency of the various diagnoses of the children with short stature

\begin{tabular}{lc}
\hline Diagnosis & No. of subjects (\%) \\
\hline Normal variants & $159(44.7)$ \\
Familial short stature & $82(23.0)$ \\
CGD & $63(17.7)$ \\
Familial short stature and CGD & $14(3.9)$ \\
Pathological short stature & $193(54.2)$ \\
Uncategorized & $4(1.1)$ \\
Total & $356(100)$
\end{tabular}

CGD, constitutional delay in growth.
Table 3. Frequency of the various diagnoses of the children with pathological short stature

\begin{tabular}{lc}
\hline Diagnosis & No. of subjects (\%) \\
\hline Growth hormone deficiency & $75(38.9)$ \\
Idiopathic short stature & $45(23.2)$ \\
Small for gestational age & $22(11.3)$ \\
Turner syndrome & $18(9.3)$ \\
Congenital heart disease & $6(3.1)$ \\
Noonan syndrome & $4(2.1)$ \\
Anemia & $4(2.1)$ \\
Hypochondroplasia & $3(1.5)$ \\
Rickets & $3(1.5)$ \\
Russel-Silver syndrome & $2(1.0)$ \\
Cancer & $2(1.0)$ \\
Precocious puberty & $2(1.0)$ \\
Scoliosis & $1(0.5)$ \\
Skeletal dysplasia & $1(0.5)$ \\
Mitochondrial cytopathy & $1(0.5)$ \\
Chronic renal failure & $1(0.5)$ \\
Hypothyroidism & $1(0.5)$ \\
DM & $1(05)$ \\
Choledochal cyst & $1(0.5)$ \\
Total & $193(100)$
\end{tabular}

DM, diabetes mellitus.

Table 4. Causes of short stature in patients with growth hormone deficiency (GHD)

\begin{tabular}{lc}
\hline Diagnosis & No. of subjects (\%) \\
\hline Idiopathic GHD & $54(72.0)$ \\
Organic GHD & $21(28.0)$ \\
Rathke's cleft cyst & $7(9.3)$ \\
Hypoxic brain damage & $7(9.3)$ \\
Small size of pituitary gland & $3(4.0)$ \\
Pituitary tumor & $2(2.7)$ \\
Microcephaly & $1(1.3)$ \\
Arnold chiari malformation & $1(1.3)$ \\
Total & $75(100)$ \\
\hline
\end{tabular}

GHD, growth hormone deficiency.

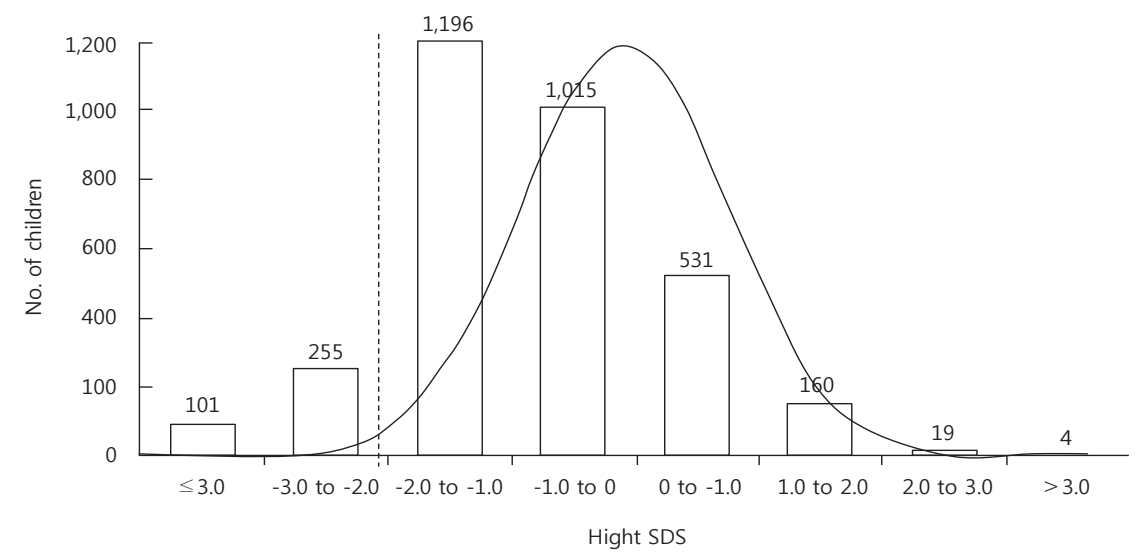

Fig. 1. Distribution of height standard deviation score (SDS) of the children with chief complaint of short stature. 
followed by small pituitary glands (4.0\%), pituitary tumors (2.7\%), microcephaly (1.3\%), and Arnold-Chiari malformation (1.3\%).

\section{Auxological characteristics of the patients with FSS, CGD and pathological short stature}

Children with FSS showed the least bone age (BA) delay, the lowest MPH SDS, normal growth velocity and the highest IGF1 level, whereas CGD children had the lowest BMI, the most BA delay, normal growth velocity, and the highest PAH SDS. Children with pathological short stature were significantly shorter, and had the lowest height velocity, height velocity SDS and IGF-I level (Table 5).

\section{Frequency of the various diagnoses according to severity of short stature}

The patients were categorized according to the severity of their short stature in two groups, that is those with a height SDS of $\leq-3$ and those with a height SDS of $>-3$. A total of 101 (43 boys and 58 girls) (28.4\%) presented very short stature; that is, their height SDS was $\leq-3$, and 255 patients (129 boys and 126 girls) $(71.6 \%)$ presented less severe short stature; that is, a height SDS of $>-3$. Even though it was not significant statistically, the most common diagnosis in the very short children was GHD (20.8\%), whereas the most common diagnosis in the less severely short children was FSS (25.1\%). CGD is more common in less severely short children than in very short children, while clinically defined syndromes are significantly more common in very short children than in less severely short children (Table 6).

\section{Discussion}

In this study, we presented auxological characteristics and distributions of various diagnoses of short stature in children who visited a pediatric endocrinology clinic due to short stature in a 3-year period. The majority of the children who visited the outpatient clinic had normal heights, while only $10.6 \%$ of the children were identified as having short stature. The percentage of patients with normal heights in this study (89.4\%) was higher than in a previous Korean report by Kim and Yoo ${ }^{11)}(62.2 \%)$. This finding suggests that more children who visited hospital with normal heights, which may suggest that the popular stereotypical preference for tall statures has intensified, or simply reflect the differences in hospital characteristics or locations, even though both involved the same tertiary hospital. In fact, $76.1 \%$ of the patients with normal heights were below the 50th percentile, suggesting that shorter patients within the normal range tended to visit outpatient clinics more often.

In the short stature group in our study, the percentage of patients with pathological short stature (54.2\%) was higher than that of patients with normal variants (44.7\%). Previous studies ${ }^{11-15)}$ have demonstrated that most children with short stature are normal variants, and may constitute more than $65 \%$ of short stature children. The reason for the discrepancy between this study and others is unclear, however, there is a

Table 6. Diagnosis according to severity of short stature

\begin{tabular}{lccr}
\hline Diagnosis & Height SDS $\leq-3$ & Height SDS $>-3$ & $P$-value \\
\hline Familial short stature & $18(17.8)$ & $64(25.1)$ & 0.142 \\
CGD & $9(8.9)$ & $54(21.2)$ & 0.006 \\
Familial short stature + CGD & $6(5.9)$ & $8(3.1)$ & 0.220 \\
Growth hormone deficiency & $21(20.8)$ & $54(21.2)$ & 0.936 \\
Idiopathic short stature & $15(14.9)$ & $30(11.8)$ & 0.429 \\
Clinically defined syndromes & $15(14.9)$ & $9(3.5)$ & $<0.001$ \\
Small for gestational age & $8(7.9)$ & $14(5.5)$ & 0.391 \\
Disorders in specific systems & $2(2.0)$ & $12(4.7)$ & 0.365 \\
Skeletal dysplasias & $4(4.0)$ & $4(1.6)$ & 0.230 \\
Endocrine disorders & $1(1.0)$ & $3(1.2)$ & 1.000 \\
(non-GHD) & & & \\
Others & $2(2.0)$ & $3(1.2)$ & 0.625 \\
Total & $101(100)$ & $255(100)$ & \\
Values are presented as numb & &
\end{tabular}

Values are presented as number (\%).

SDS, standard deviation score; CGD, constitutional delay in growth; GHD, growth hormone deficiency.

Table 5. Auxological characteristics of children with normal variant and pathological short stature

\begin{tabular}{|c|c|c|c|c|}
\hline Variable & FSS $(n=82)$ & $\operatorname{CGD}(n=63)$ & Pathologic SS ( $n=193)$ & $P$-value \\
\hline Age (yr) & $10.21 \pm 5.28$ & $8.23 \pm 3.63$ & $7.42 \pm 3.90$ & $<0.001$ \\
\hline Sex, male/female & $24 / 58$ & $38 / 25$ & $103 / 90$ & $<0.001$ \\
\hline Height SDS & $-2.66 \pm 0.58$ & $-2.51 \pm 0.45$ & $-2.92 \pm 0.85$ & $<0.001$ \\
\hline Body mass index $\left(\mathrm{kg} / \mathrm{m}^{2}\right)$ & $18.01 \pm 3.39$ & $15.75 \pm 2.64$ & $16.33 \pm 2.86$ & $<0.001$ \\
\hline Bone age delay (yr) & $0.50 \pm 0.88$ & $1.80 \pm 0.72$ & $1.73 \pm 3.12$ & $<0.001$ \\
\hline Height velocity (cm/yr) & $4.47 \pm 2.51$ & $4.78 \pm 1.27$ & $3.92 \pm 1.83$ & 0.006 \\
\hline Height velocity SDS & $0.07 \pm 1.70$ & $-0.32 \pm 1.49$ & $-1.14 \pm 1.70$ & $<0.001$ \\
\hline MPHSDS & $-2.09 \pm 0.39$ & $-0.57 \pm 0.64$ & $-0.67 \pm 0.97$ & $<0.001$ \\
\hline PAHSDS & $-3.38 \pm 5.40$ & $-0.78 \pm 1.13$ & $-2.56 \pm 0.37$ & 0.004 \\
\hline IGF-I (ng/mL) & $208.38 \pm 17.97$ & $161.30 \pm 82.92$ & $135.23 \pm 83.21$ & $<0.001$ \\
\hline IGFBP-3 ( $\mu \mathrm{g} / \mathrm{mL})$ & $2.24 \pm 0.58$ & $2.15 \pm 0.65$ & $2.02 \pm 0.70$ & 0.086 \\
\hline
\end{tabular}

Values are presented as mean \pm standard deviation.

FSS, familial short stature; CGD, constitutional delay in growth; SS, short stature; SDS, standard deviation score; MPH, midparental height; $\mathrm{PAH}$, predicted adult height. 
possibility of referral bias in tertiary hospitals.

In the pathological short stature group in this study, GHD was the most common cause of short stature (38.9\%), followed by ISS $(23.2 \%)$, small for gestational age (11.3\%), Turner syndrome $(9.3 \%)$, and others. High prevalence of GHD as a cause of short stature has been reported in almost all previous studies. In our study, GHD was diagnosed in approximately $20 \%$ of the children with short stature, which is similar to the $22.8 \%$ in Zargar et al. ${ }^{15)}$. and the $23.4 \%$ in Moayeri and Aghighi $^{16)}$. In contrast, other studies ${ }^{11-13)}$ showed GHD occurred in $11.1 \%-13.0 \%$ of short stature cases, which is less than in our study. Even though most GHD is idiopathic, 28\% of GHD cases revealed organic abnormalities, such as Rathke's cleft cysts ${ }^{17)}$, small-sized pituitary glands, pituitary tumors or other anatomic abnormalities, suggesting that comprehensive evaluations for combined morbidities or anatomical abnormalities are required for patients with GHD.

As expected, the comparison of the auxological characteristics of FSS, CGD, and pathological short stature confirmed these findings: (1) The height velocity and height velocity SDS are lowest in pathological short stature; (2) the bone age delay is lowest in FSS; (3) the MPH SDS is lowest in FSS; (4) the PAH SDS is normal in CGD; (5) the IGF-I level is lowest in pathological short stature.

The heights of those with pathological short stature were shorter than those for normal variants; therefore, physicians should evaluate the possibility of pathologic diseases more carefully when the patients have lower height SDS within the short stature group. In our study, children with a very short stature (that is, height SDSs of $\leq-3$ ) were more likely to have pathological short stature. Normal variants were present in about $49.4 \%$ of children with less severe short stature (that is, height SDSs of $>-3$ ), while in only $32.6 \%$ of the very short children. These results are comparable with a previous report by Papadimitriou et al. ${ }^{13)}$ that also showed there were more normal variants in less severe groups than in very short stature ones.

In conclusion, a careful monitoring of growth and a precise evaluation of short stature are very important to discover the pathologic causes and determine whether GH treatment is needed ${ }^{18)}$. Our data shows that only $10.6 \%$ of the children with chief complaint of short stature were identified as having short stature. Approximately 54\% of children of short stature presented pathological short stature, whereas normal variants were diagnosed in about $45 \%$ of the short children. GHD was the most common cause of pathological short stature, and was found in approximately $20 \%$ of the children with short stature.

\section{Conflict of interest}

No potential conflict of interest relevant to this article was reported.

\section{References}

1. Rieser PA. Educa1. Rieser PA. Educational, psychologic, and social aspects of short stature. J Pediatr Health Care 1992;6(5 Pt 2):325-32.

2. Lechelt EC. Occupational affiliation and ratings of physical height and personal esteem. Psychol Rep 1975;36:943-6.

3. Gordon M, Crouthamel C, Post EM, Richman RA. Psychosocial aspects of constitutional short stature: social competence, behavior problems, self-esteem, and family functioning. J Pediatr 1982;101:477-80.

4. Stabler B, Clopper RR, Siegel PT, Stoppani C, Compton PG, Underwood LE. Academic achievement and psychological adjustment in short children. The National Cooperative Growth Study. J Dev Behav Pediatr 1994;15:1-6.

5. Sandberg DE, Voss LD. The psychosocial consequences of short stature: a review of the evidence. Best Pract Res Clin Endocrinol Metab 2002;16:449-63.

6. Kranzler JH, Rosenbloom AL, Proctor B, Diamond FB Jr, Watson M. Is short stature a handicap? A comparison of the psychosocial functioning of referred and nonreferred children with normal short stature and children with normal stature. J Pediatr 2000;136:96-102.

7. Allen DB, Cuttler L. Clinical practice. Short stature in childhood--challenges and choices. N Engl J Med 2013;368:1220-8.

8. Ranke MB. The Kabi Pharmacia International Growth Study: aetiology classification list with comments. Acta Paediatr Scand Suppl 1991;379:87-92.

9. Greulich W, Pyle S. Radiographic atlas of skeletal development of the hand and wrist. 2nd ed. Stanford: Stanford Iniversity Press, 1959.

10. Bayley N, Pinneau SR. Tables for predicting adult height from skeletal age: revised for use with the Greulich-Pyle hand standards. J Pediatr 1952;40:423-41.

11. Kim JB, Yoo HW. Etiological classifications of children with chief complaint of short stature. J Korean Soc Pediatr Endocrinol 1997;2:1-9.

12. Lashari SK, Korejo HB, Memon YM. To determine frequency of etiological factors in short statured patients presenting at an endocrine clinic of a tertiary care hospital. Pak J Med Sci 2014;30:858-61.

13. Papadimitriou A, Douros K, Papadimitriou DT, Kleanthous K, Karapanou O, Fretzayas A. Characteristics of the short children referred to an academic paediatric endocrine clinic in Greece. J Paediatr Child Health 2012;48:263-7.

14. Lindsay R, Feldkamp M, Harris D, Robertson J, Rallison M. Utah Growth Study: growth standards and the prevalence of growth hormone deficiency. J Pediatr 1994;125:29-35.

15. Zargar AH, Laway BA, Masoodi SR, Wani AI, Salahuddin M. An aetiological profile of short stature in the Indian subcontinent. J Paediatr Child Health 1998;34:571-6.

16. Moayeri H, Aghighi Y. A prospective study of etiology of short stature in 426 short children and adolescents. Arch 
Iranian Med 2004;7:23-7.

17. Briceno LG, Gunczler P, Solis O. Rathke cleft cyst as cause of growth hormone deficiency in a 9-year-old girl. J Pediatr 2012;160:708-708.e1.
18. Rogol AD, Hayden GF. Etiologies and early diagnosis of short stature and growth failure in children and adolescents. J Pediatr 2014;164(5 Suppl):S1-14.e6. 